\title{
الرحالة والجغرافيون في \\ أوروبا العصور الوسطى
}

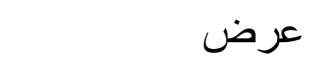

$$
\begin{aligned}
& \text { د. هانم عبد الرحيم إيراهيم } \\
& \text { أستاذ المكتبات و المعلومات } \\
& \text { المساعد بجامعة الإسكندرية }
\end{aligned}
$$

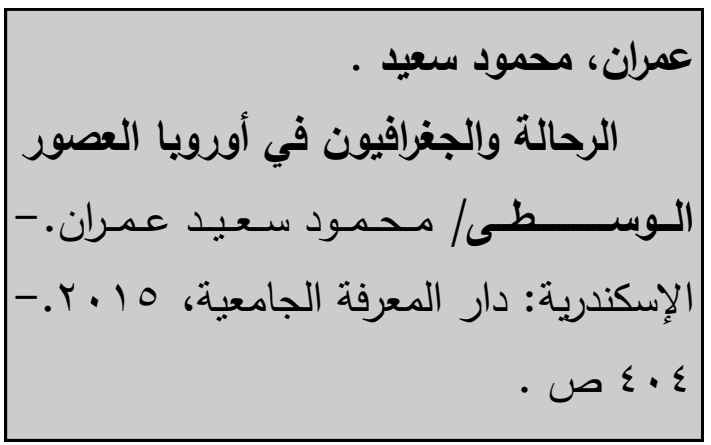


الوسطى، بينما تتاول الباب الثاني الجغرافيين

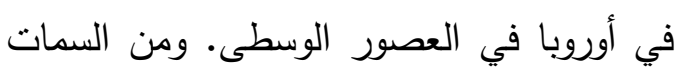
المميزة لهذا العمل أنه يتضمن عددًا من بحوث الثرن المؤتمرات التي تقدم بها المؤلف للجهات العلمية المتخصصة في المجال. وقد ألحق بنهابة هذا الكتاب قائمة بمختصرات أسماء المصادر والمراجع الإنجليزية المستخدمة بالكتاب، بالإضافة إلي أربع قوائم للمصادر والمراجع التي تم الاستشهاد بها في هذا الكتاب ؛ وشملت قائمة المصادر الأجنبية TV TV مصدرًا باللغة

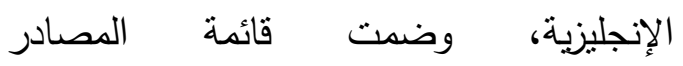
العربية 17 مصدرًا باللغة العربية، بينما اشتملت

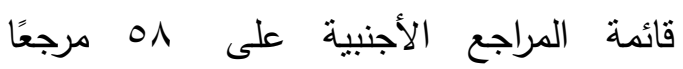
بالإنجليزية، أما قائمة المراجع العربية والمعربة فتضمنت سا مرجعًا بالعربية. يرجع أقدم هذه

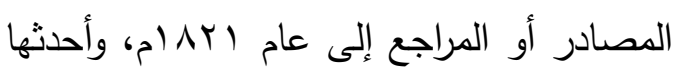

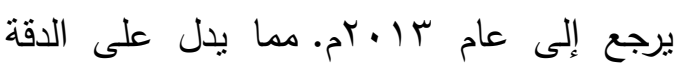

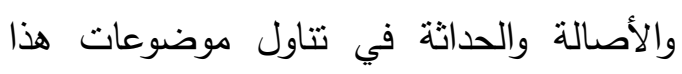

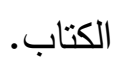

رغم أن هذا العمل موجه أساسًا لطلاب

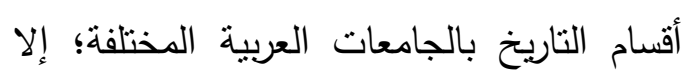
أن هناك عددًا من المستفيدين المحتملين الذين قد يهمهم التعرف بالتقصيل على هذا التخصص فن هن الدقيق المتميز الذي تم تتاوله في هذا الكتاب، وهو موضوع الرحالة والجغرافيين الأجانب الذين

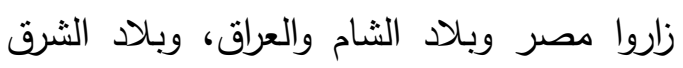
في خلال العصور الوسطى الأوروبية. ومن بين
يعتبر هذا العمل هو أول كتاب يوضع في العالم العربي عن موضوع الرحالة والجغرافيين في أوروبا في العصور الوسطى، وقام بإعداد هذا الكتاب المتميز، الراحل الأستاذ

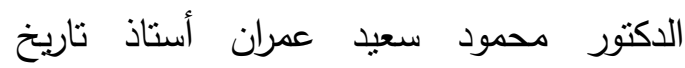
وحضارة أوروبا في العصور الوسطى بكلية

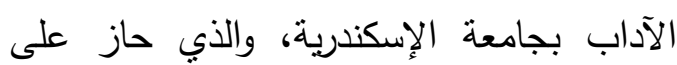
جوائز الجامعة التقديرية والتشجيعية والتميز العلمي، نظرًا لعطائه العلمي وتميزه في مجال

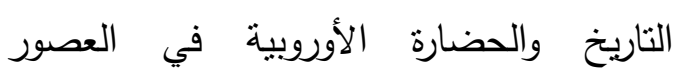
الوسطي. وكان هذا العالم الراحل هو المرشح المصري الوحيد لنيل جائزة بالزان العالمية في

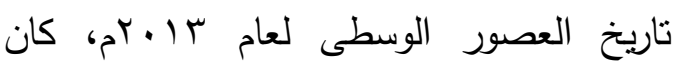
سيادته - رحمه الله - عضوًا بارزًا في الجمعيات المحلية والإقليمية والعالمية في لياتية المجال، ومنها جمعية الحروب الصليبية والثرق اللاتييني. وقد قام الأستاذ الراحل بإعداد ستة وستين بحثًا، واثثين وعشرين كتابًا في تاريخ وحضارة أوروبا في العصور الوسطى. كما ولنين وتنسين أثرف سيادته على تسع وثثلاثين أطروحة للماجستير والدكتوراه ، بالإضافة إلى مشاركته الفعالة في المؤتمرات المحلية والعالمية. تعتبر هذه الأبحاث العلمية ذات قيمة علمية متميزة بما تحمله من دقة البحث والابتكار، والإضافات الجديدة للعلم ينقسم هذا الكتاب الشيق إلى بابين: تتاول الباب الأول الرحالة في أوروبا في العصور 
بهذا الإنتاج وتساعد الباحث العربي في الحصر الببليوجرافي لكتب الرحالة والجغرافيين الأجانب للتعرف على السمات الموضوعية التي تتاولتها كتب الرحالة الأجانب، وكذلك التقبمات الموضوعية لعلم الجغرافيا في ذلك الوقت، كما يمكن من خلاله متابعة مصادر المعلومات التي اعتمد عليها هؤلاء الجغرافيون الأجانب ومدى من هئه تواصلهم مع الجغرافيين العرب، والمسلمين في هولاه ذلك الوقت.

تتاول الباب الأول من هذا الكتاب تسعة مجالات خاصة بالرحالة الأوروبيين في العصور الوسطى، تمثلت هذه المجالات فيما يلي:

1- مصر والثام في كتب بعض الرحالة الأجانب في العصر البيزنطي، قد نم التقدم بهذا البحث إلى مؤتمر الإسكندرية الدولي الأول حول التبادل الحضاري بين شعوب الإني البحر المتوسط عبر التاربخ، وقد عقد هذا المؤتمر في الفترة من بr-Y ب بناير عام ع 99 ام، وذلك في كلية الآداب بجامعة

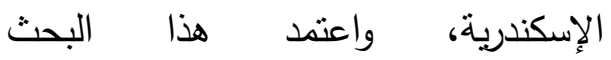
على التسجيلات التي خلفها ثلاثة من الرحالة الأجانب الذين زاروا مصر والثام بعد ظهور المسيحية ؛ حيث كانت الرحلات التي تتم خلال ذلك الوقت من الرحلات الدينية التي يقوم بها من يطلق

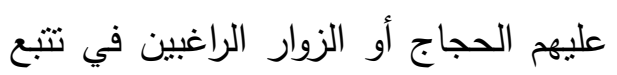

هذه الفئات التي يمكنها الإفادة من هذا العمل الجغرافيون المرتمون بالجغرافيا التاريخية، وكذللك الباحثون المهتمون بالخرائط القديمة للمنطقة العربية أو في العالم القديم خاصة تلاك التي رسمها الجغرافيون الأوربيون الأوائل؛ حيث يشتمل هذا الكتاب على بيانات عن ؟ب خريطة جغرافية قام برسمها الجغرافيون الأجانب منذ القرن السادس قبل الميلاد مرورًا بالعصور

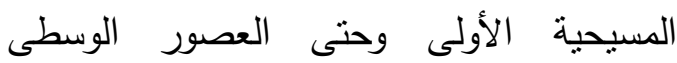

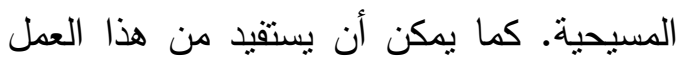
أيضًا المرتمون بالكتابة عن أدب الرحلات وكتب الرحالة الأجانب في تلاك العصور، وهذا المجال من المجالات المتميزة التي لم يتم تتاولها من جانب الباحثين في عالمنا العربي، بالإضافة إلى ذلك يعتبر هذا العمل مصدرًا هامًا للباحثين بالين في مجال المكتبات والمعلومات المهتمين بحصر الإنتاج الفكري الأجنبي عن المنطقة العربية في العصور الوسطى ؛ وهذه الفترة الزمنية بالذات تم تتاولها من جانب عدد من الباحثين، ولكن من خلال حصر الإنتاج الفكري العربي لعلماء المسلمين في عصور الحضارة الإسلامية التي عاصرت العصور الوسطى لئى بأوروبا، أما الإنتاج الفكري الأجنبي الذي تتاول موضوعات خاصة بالمنطقة العربية أو العالم الإسلامي فلم يتم حصره، وبحث خصائصه من بن

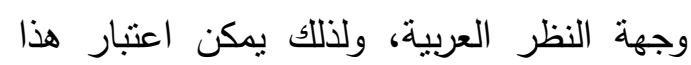
الكتاب أحد المصادر العربية الهامة التي تعرف اعبهر 
تفاصيل عن بيت المقدس في عهز معاوية ابن أبي سفيان، وموقع المدينة، وبواباتها ومسجدها، وكنائسها. وتتابعت تفاصيل هذه ونه الرحلة في دمشق والإسكندرية، وكريت ثم وماندان القسطنطينية، واشتملت هذه التفاصيل على ولى معلومات هامة عن النواحي الاقتصادية، والاجتماعية، والمعمارية والحضارية في ذلك الك الكيه

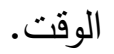

r- استعرض المؤلف في هذا البحث " القدس والمسجد الأقصى في كتابات الرحالة

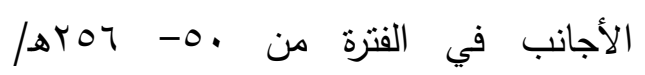
. عصور الحضارة الإسلامية، وقد ألقي هذا البحث في جامعة دمشق التي نظمت

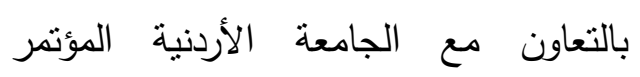
الدولي الثامن حول تاريخ بلاد الثام في لإني

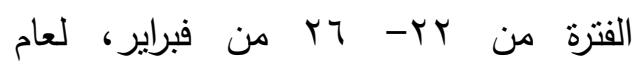

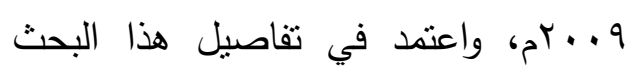
على المصادر الغربية، وعلى التوراة فقط دون الرجوع للنص القرأني، لأن هؤلاء الرحالة لايؤمنون بما جاء في القرآن، وعلى لانى ذلك تم سرد التفاصيل التي وردت بتلك

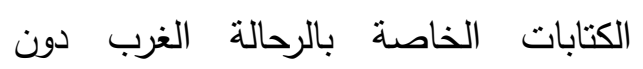
مقارنتها بما جاء بالقرآن، والتي من خلالها يمكننا التعرف على طرق ومناهج الفكر باء بان الغربي والمغالطات التاريخية التي دونت في كتبهم
خطوات الأنبياء، ومشاهدة الأماكن التي وقعت فيها المعجزات في بيت المقد، وفي مصر، ولذلك فقد تم التركيز على التسجيلات التي خلفها كل من القديسة سلفيا أكويتين، والقديسة بولا والقديس جيروم الذي صاحبها في بعض أجزاء من هذه الرحلة، والتي قام جيروم بتسجبل بياناتها بدلا من القديسة بولا التي لم تدون بنفسها

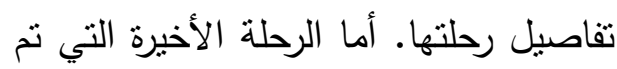
تتاولها في هذا البحث فهي تلك التي قام بها الرحالة ثيودوسيوس. وقد تضمنت هذه

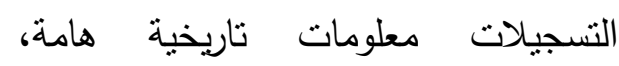
وتفصيلات خاصة بالأماكن التي تم زيارتها ووصف للمدن والحياة الاجنماعية والأشجار والجبال وغيرها من التقاصيل الخاصة بالأماكن المقدسة.

ץ- تتاول المجال الثاني من مجالات هذا

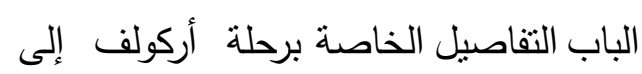

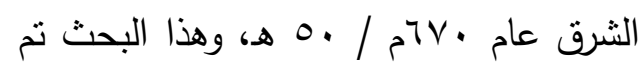

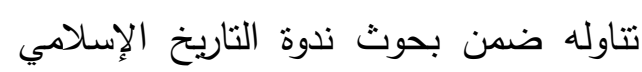
والوسيط المنعقدة في جامعة عين شمس عام

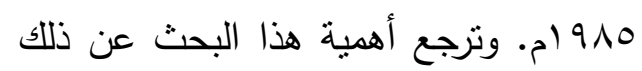
الرحالة أن أركولف يعتبر أول رحالة غربي الهيه زار الأماكن المقدسة، وبعض المناطق الشرقية عقب الفتح الإسلامي. تلأك الفترة التي لم تصلنا عنها تسجيلات باللغة العربية. وتتاولت تشجيلات رحلة أركولف بلت بالفه 
العدد الرابع والخمسـون - 7 •

الكتاب؛ وفيه تتاول المؤلف الرحلات التبثيرية التي قام بها مبعوثون من قبل البابا لبلاد الثرق والأمبراطورية المغولية،

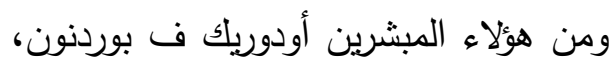
والذي أرسل هناك لإعداد مراكز تبشيرية للديانة المسيحية للمذهب الكاثوليكي. - V الأوربيين هو رحلة جوردانوس القطلاني، ومن النتائج التي نتجت عن هذه الرحلة كتاب وصف عجائب الثرق الذي يتتاول الفترة من وץسا - یrسام ، وتحدث فيه عن المعلومات الخاصة بالبحر المنوسط،، وأرمينيا وبلاد قارس، وبلاد الهند. 1- البحث الثامن في هذا الباب تتاول مصر والثام في كتابات الرحالة والجغرافي يوحنا

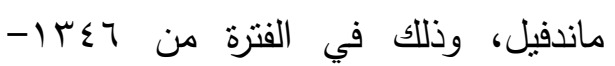
\&V الوطن العربي عبر العصور الذي انعقد

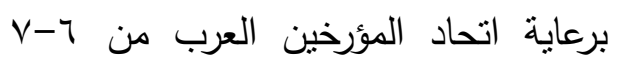
نوفمبر عام rا •Yم، ونشر في حصاد اتحاد المؤرخين العرب لعام با ـ ب م.

9- أما البحث التاسع والأخير في هذا الباب فتضمن تفاصيل رحلة أفاناسي نيكتين الروسي الرب رحلته إلى بحر قزوين، والمحيط الهندي (البحر العربي)، بالإضافة إلى البحر ولى
ع- كانت رحلة الثهيد أنطونيوس إلى بلاد

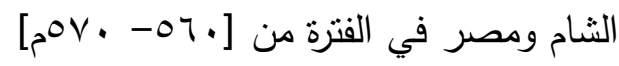
هي رابع مجالات البحث في هذا الباب الأول، وتم التقام بها البحث إلى لهى ندوة العلاقات بين الثرق والغرب في العصور الوسطى .ب أكتوبر - 1 - نوفمبر لعام ب99 ام بكلية الآداب بجامعة المنيا، ونشر أيضًا في مجلة التاريخ والمستقبل، كلية

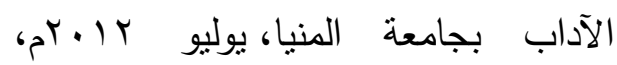
وتتاول هذا البحث الأديان، والأديرة، والمخلفات المقدة، والتعاويذ، والمعلومات الجغرافية، الاقتصادية، والحيوانات، وتحصينات المدن والتكايا

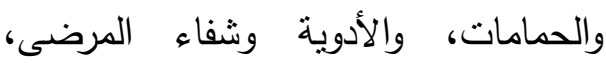
والتعليم واللغة، والعادات الاجتماعية. 0- تم في هذا البحث الخامس تتاول أسطورة الكاهن يوحنا ودورها في تحالف الصليبيين

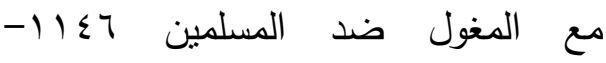
| (ام، وقد ألقي هذا البحث في مؤتمر الدين والسياسة في الوطن العربي عبر العصور، الذي نظمه المجلس الأعلى للافقة والجمعية المصرية للدراسات

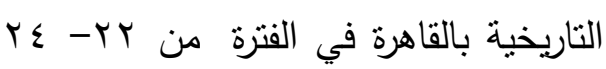

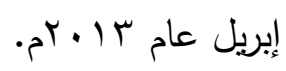

7- البعثات التبثيرية إلى الثرق هي المبحث

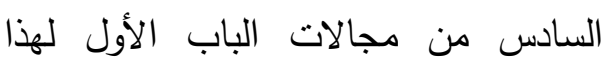




\section{الفهرست}

الجغرافيين القدماء في العصور الوسطى.

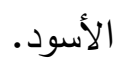

وأبدى المؤلف ملاحظاته على هذه الخرائط الجغرافية الكلاسيكية.

ويتضح من خلال استعراض هذا الكتاب

المتميز مدى الأصالة والجدة في نتاوله

المجالات المختلفة الخاصة بالرحالة والجغرافيين

في العصور الوسطى بأوروبا، والتي تثري بالتي

المكنبة العربية بأحد أهم مصادر الإنتاج

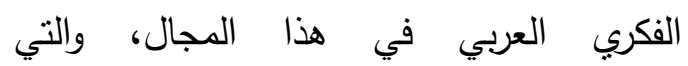

تساعد الباحثين والمشتغلين العرب بهذا المجال

في إعداد بحوث جديدة متميزة.

هذا الباب فتتمنل فيما يلي:

$$
\begin{aligned}
& \text { ا ـ الجغرافيون، وبدايات العصور الوسطى } \\
& \text { r. إيسدور الإشبيلي. } \\
& \text { r. الجغرافيون المسلمون. }
\end{aligned}
$$

ع. عصر النهضة وأثزه على العلوم الجغرافية

$$
\text { في أوروبا. }
$$

ه. نشاط البرتغاليين عبر البحار والطرق

$$
\text { التجارية. }
$$

7. المدرسة الألمانية الجغرافية.

V. مدرسة رسم الخرائط الفلمنكية (هولندا

وبلجيكا) والإنجليزية . 100- .010 (م.

^. الخرائط الجغرافية في أوروبا العصور

الوسطى : دراسة تاريخية، وهذا هو لهرئه

المبحث الأخير في هذا الكتاب والذي لوني

تضمن تفاصيل عن علم الخرائط، وأورد

من خلاله عس خريطة جغرافية من أعمال 